\title{
Relation of Dissociative Phenomena to Levels of Cerebrospinal Fluid Monoamine Metabolites and Beta- Endorphin in Patients With Eating Disorders: A Pilot Study
}

\author{
Mark A. Demitrack, Frank W. Putnam, David R. Rubinow, Teresa A. Pigott, \\ Margaret Altemus, Dean D. Krahn, and Philip W. Gold
}

Received December 28, 1992; revised version received May 4, 1993; accepted June 24, 1993.

\begin{abstract}
Dissociation is made manifest by a failure to integrate thoughts, feelings, memories, and actions into a unified sense of consciousness. Although dissociation is presumed to be a special state of consciousness manifested by state-dependent memory and physiology, the psychobiology of dissociation is poorly understood. In this study, we examined cerebrospinal fluid levels of the major monoamine metabolites and $\beta$-endorphin in patients with eating disorders (11 with anorexia nervosa, 16 with bulimia nervosa), while they were acutely ill. Dissociative capacity was measured using the Dissociative Experiences Scale (DES). We provide evidence that neurochemical changes in dopaminergic, serotonergic, and opioid systems may be associated with the clinical expression of dissociation in patients with eating disorders during the acute phase of their illness. These preliminary results are compatible with previous studies of neurochemical disturbances in the eating disorders and suggest that future work in dissociation should specifically include examination of these neurobiologic systems.
\end{abstract}

Key Words. Dissociation, hypnotizability, anorexia nervosa, bulimia, homovanillic acid, 5-hydroxyindoleacetic acid.

Dissociation is a complex psychobiological event which is evidenced by a failure of the normally integrative functions of identity, memory, and consciousness. Although it is generally thought to exist along a continuum of severity, dissociation, in its more extreme forms, may be characterized by severe disturbances in memory (typically amnesias), as in the dissociative disorders described in DSM-III-R (American

\footnotetext{
Mark A. Demitrack, M.D., is Assistant Professor of Psychiatry and Director of the Michigan Eating Disorders Program, Department of Psychiatry, University of Michigan, Ann Arbor, MI. Frank W. Putnam, M.D., is Chief, Unit on Dissociative Disorders, Laboratory of Developmental Psychology, National Institute of Mental Health, Bethesda, MD. David R. Rubinow, M.D., is Chief, Section on Behavioral Endocrinology, Biological Psychiatry Branch, National Institute of Mental Health, Bethesda, MD. Teresa A. Pigott, M.D., is Assistant Professor of Psychiatry, Department of Psychiatry, Georgetown University Medical School, Washington, DC. Margaret Altemus, M.D., is Senior Staff Fellow, Laboratory of Clinical Science, National Institute of Mental Health, Bethesda, MD. Dean D. Krahn, M.D., is Associate Professor of Psychiatry, Department of Psychiatry, University of Wisconsin, Madison, WI. Philip W. Gold, M.D., is Chief, Clinical Neuroendocrinology Branch, National Institute of Mental Health, Bethesda, MD. (Reprint requests to Dr. M.A. Demitrack, Michigan Eating Disorders Program, University of Michigan School of Medicine, 1500 E. Medical Center Dr., Ann Arbor, MI 48109-0118, USA.)
} 
Psychiatric Association, 1987), or by gross alterations in sensorimotor function (e.g., hysteria), which often have specific psychological meaning for the individual (World Health Organization, 1978). Psychophysiological studies suggest that dissociation is a special state of consciousness manifest by state-dependent memory and physiology (Putnam, 1991b). As a group, psychiatric patients have higher levels of dissociative experience than normal subjects (Bernstein and Putnam, 1986). Furthermore, an increased capacity for dissociative experience is associated with a history of trauma and appears to contribute to an increased incidence of self-destructive behaviors in psychiatric patients (Putnam, 1985; Ross and Norton, 1989; Demitrack et al., 1990; Quimby and Putnam, 1991; Spiegel, 1991). Although there has been speculation about the neurobiological mechanisms of dissociation based on this psychophysiologic data, to date there are no studies of the relationship between dissociation and central nervous system (CNS) neurochemicals.

We have previously shown that patients with eating disorders manifest a spectrum of severity of dissociative experience, which may range as high in some individuals as that seen in patients with multiple personality disorder (Demitrack et al., 1990). Furthermore, we showed that the propensity to dissociate is itself associated with an increase in self-harmful behavior and an overall increase in affective dysphoria. We proposed that endogenous opioids and certain monoaminergic neurotransmitters may play important roles in the neurobiology of dissociative experience.

The purpose of this pilot study was to suggest potential neurobiologic substrates for future study by exploring associations between measures of dissociation and CNS neurochemicals specifically known to be disturbed in eating disorder patients. We provide preliminary data suggesting that the presence of dissociative phenomena in patients with eating disorders may be associated with alterations in central monoaminergic and opioid systems.

\section{Methods}

Subjects. Twenty-seven female patients, admitted consecutively to the Unit on Eating Disorders in the Clinical Center of the National Institutes of Health, were studied. All patients met $D S M-I I I-R$ criteria for either anorexia nervosa $(n=11)$ or bulimia nervosa $(n=16)$. No other Axis I diagnoses were present. Mean age \pm 1 SD of the patient group was $22.9 \pm 4.7$ years (age range 16-39). Mean age for anorexic patients did not differ from the mean age for bulimic patients ( $23.5 \pm 6.9$ years vs. $22.4 \pm 2.4$ years). Anorexic patients were underweight at the time of study $(62.0 \pm 5.1 \%$ of population average body weight $)$, while all bulimic patients werc within $\pm 20 \%$ of population average body weight $(92.2 \pm 10.3 \%$ ). All subjects had participated in our previous study of dissociative phenomena in eating disorders (Demitrack et al., 1990).

A group of 27 age-matched normal women served as a comparison group for the patients on the dissociation ratings. Mean age $\pm 1 \mathrm{SD}$ of this normal comparison group was $22.5 \pm 4.4$ (age range 17-38). Matching was pairwise and was successful to within 2 years of age for each patient and control subject. A separate group of 11 normal women served as a comparison group in the lumbar puncture studies. Mean age $\pm 1 \mathrm{SD}$ of the second group of normal women was $29.7 \pm 9.3$ (age range 21-50). Both sets of comparison subjects were recruited by advertisement from the Washington, D.C. area and were free of a current or past history of psychiatric illness, as assessed by an unstructured psychiatric interview. All normal female subjects were within $90-100 \%$ of population average body weight for their age and height. 
Procedure. Dissociative capacity was assessed using the Dissociative Experiences Scale (DES), a previously validated 28 -item visual analog rating scale (Bernstein and Putnam, 1986). The response format for each item on the visual analog scale consisted of a $100-\mathrm{mm}$ line with no divisions and numerically anchored at the end points. The subject was asked to indicate her response by making a slash across the line at the appropriate place. Each item score was determined by measuring the subject's slash mark to the nearest $5 \mathrm{~mm}$ from the left-hand anchor point. The total score was the average of the 28 item scores. Additional self-report measures of affective distress included the Beck Depression Inventory (BDI; Beck et al., 1961) and the State scale of the Spielberger State-Trait Anxiety Inventory (STAI; Spielberger et al., 1970).

Lumbar punctures were performed on the anorexic subjects after 3 weeks of nutritional stabilization but before any weight restoration. Bulimic subjects underwent lumbar puncture within 48 hours after admission. In all the normal subjects, and in all patients with regular menses, lumbar punctures were performed in the early follicular phase of the cycle (days 1-7). Lumbar punctures were performed in a left lateral decubitus position between 0900 and $0930 \mathrm{~h}$ with patients at strict bed rest after an overnight fast. Over $15-30$ minutes, $30 \mathrm{ml}$ of cerebrospinal fluid (CSF) were withdrawn. Monoamine metabolites were measured in an aliquot taken from the first $3 \mathrm{ml}$, while $\beta$-endorphin was determined in an aliquot taken from a pool representing the $15 \mathrm{th}$ to $27 \mathrm{th} \mathrm{ml}$. Samples were collected in chilled polyethylene tubes, immediately frozen on dry ice after collection, and then stored at $-70^{\circ} \mathrm{C}$ until assayed.

The norepinephrine metabolite 3-methoxy-4-hydroxyphenylglycol (MHPG), the dopamine metabolitc homovanillic acid (HVA), and the serotonin metabolite 5-hydroxyindoleacetic acid (5-HIAA) were measured by means of high-performance liquid chromatography with electrochemical detection (Seppala et al., 1984). Intra-assay and interassay variabilities ranged from $5.6 \%$ to $14.7 \%$ and $4.6 \%$ to $5.8 \%$, respectively. A radioimmunoassay (INCSTAR, Stillwater, $\mathrm{MN}$ ) was used to measure $\beta$-endorphin. The sensitivity for the $\beta$-endorphin assay was $1 \mathrm{fmol} / \mathrm{ml}$, and the intra-assay and interassay variabilities were $6.2 \%$ and $19.8 \%$, respectively.

Since previous analyses have shown that scores on the DES are not normally distributed (Bernstein and Putnam, 1986), the Wilcoxon signed-ranks test was used to determine differences between paired DES scores for patients and normal subjects, while the Spearman rank order correlation coefficient was used to assess the degree of association between behavioral scores and the pertinent biological measures. Differences among group mcans for the biological variables were determined by one-way analysis of covariance, with correction for unequal variances when appropriate, and with age as a covariate. Where a significant difference among means was noted, post hoc comparisons were performed with the StudentNewman-Keuls multiple range test. All statistical comparisons were two-tailed. Analyses were performed on a Macintosh SE/30 microcomputer using the Statview 512+ statistical software package (BrainPower, Inc., Calabasas, CA) and on an Amdahl $470 \mathrm{~V} / 8$ mainframe system using the BMDP statistical package (BMDP Statistical Software, Inc., Los Angeles, CA).

\section{Results}

Table 1 presents summary data for total scores on the DES and for CSF levels of the monoamine metabolites and $\beta$-endorphin. Since nonparametric statistics were used for their analysis, all DES scores are presented as median values. All biological indices are presented as the mean $\pm 1 \mathrm{SD}$, along with the adjusted group means used in the analyses of covariance. For the correlational analyses, data are presented for the entire patient group, as well as for the separate diagnostic categories (Table 2).

Relation Between CSF Neurochemicals and Nutritional Status. Consistent with reports by others (Kaye et al., 1987, 1988), we found a significant weightassociated reduction in CSF levels of both 5-HIAA and $\beta$-endorphin in the group of underweight anorexic patients compared with the corresponding levels in either the 


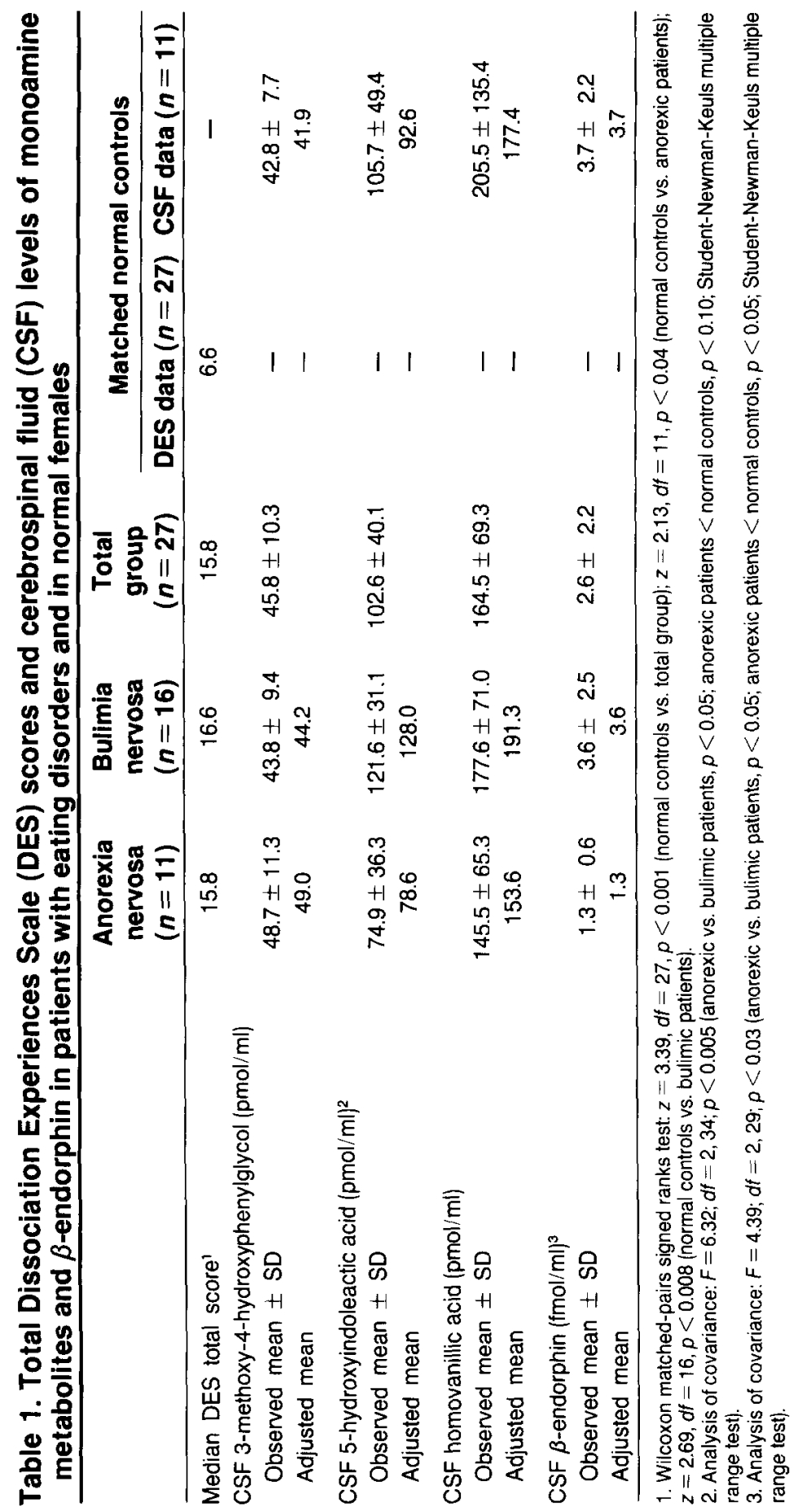


Table 2. Spearman rank-order correlation coefficients for scores on the Dissociative Experiences Scale (DES), the Beck Depression Inventory (BDI), and the State Scale of the Spielberger State-Trait Anxiety Inventory (STAI) and cerebrospinal fluid levels of monoamine metabolites and $\beta$ endorphin in patients with eating disorders

\begin{tabular}{lccc}
\hline & DES & BDI & STAI \\
\hline Total eating disorder group correlations $(\boldsymbol{n}=\mathbf{2 7})$ & & & \\
3-Methoxy-4-hydroxyphenylglycol & 0.17 & 0.25 & 0.33 \\
5-Hydroxyindoleacetic acid & 0.21 & -0.19 & 0.12 \\
Homovanillic acid & $0.41^{1}$ & 0.13 & 0.33 \\
$\beta$-endorphin & $-0.44^{1}$ & $0.53^{1}$ & $-0.48^{1}$ \\
Bulima nervosa group correlations $(\boldsymbol{n}=\mathbf{1 6})$ & & & \\
3-Methoxy-4-hydroxyphenylglycol & & & \\
5-Hydroxyindoleacetic acid & 0.37 & 0.20 & 0.35 \\
Homovanillic acid & $0.62^{1}$ & 0.09 & 0.43 \\
$\beta$-endorphin & $0.51^{2}$ & 0.25 & $0.58^{1}$ \\
Anorexia nervosa group correlations $(\boldsymbol{n}=\mathbf{1 1})$ & $-0.63^{1}$ & -0.41 & -0.51 \\
3-Methoxy-4-hydroxyphenylglycol & & & \\
5-Hydroxyindoleacetic acid & -0.20 & 0.09 & 0.27 \\
Homovanillic acid & -0.08 & -0.13 & 0.10 \\
$\beta$-endorphin & 0.18 & -0.02 & 0.11 \\
\hline
\end{tabular}

Note. In one of the anorexic subjects, and in two of the bulimic subjects, $\beta$-endorphin values were unavailable.

1. $p<0.05$, two-tailed test.

2. $p=0.05$, two-tailed test.

normal-weight patients with bulimia nervosa or the normal female subjects (Table 1). Furthermore, in the overall group of patients, the percent of population average body weight, a general measure of illness severity and nutritional compromise, showed a significant positive correlation with CSF levels of both 5-HIAA (Spearman's $\rho=0.64, d f=27, p<0.01$ ) and $\beta$-endorphin (Spearman's $\rho=0.43$, $d f=24, p<0.05$ ), as well as a trend toward a positive correlation with CSF levels of HVA (Spearman's $\rho=0.37, d f=27, p<0.10$ ).

Relation Between CSF Neurochemicals and Behavioral Measures. In the overall patient group, there was a significant positive correlation between the total DES score and CSF levels of HVA (Spearman's $\rho=0.41, d f=27, p<0.05$ ) (Table 2 ). On the other hand, CSF levels of $\beta$-endorphin were inversely associated with total DES scores (Spearman's $\rho=-0.44, d f=24, p<0.05$ ) (Table 2). To assess the specificity of the relation between total DES scores and CSF levels of HVA and $\beta$-endorphin, the relation between BDI and STAI scores and the CSF neurochemicals was also examined. While the levels of CSF $\beta$-endorphin also correlated inversely with BDI and STAI scores, CSF HVA was not significantly related to either index of behavioral distress (Table 2).

Correlational analyses were also performed for the separate diagnostic categories (see Table 2). Of particular note, there were no significant associations between any of the behavioral or biological measures in the group of underweight anorexic 
subjects ( $n=11)$. In contrast, normal-weight patients with bulimia nervosa $(n=16)$ showed a significant positive association between total DES score and either HVA (Spearman's $\rho=0.51, d f=16, p=0.05$ ) or 5-HIAA (Spearman's $\rho=0.62, d f=16$, $p<0.02$ ), and an inverse association with $\beta$-endorphin (Spearman's $\rho=-0.63$, $d f=14, p<0.03$ ). Levels of HVA in CSF were also positively associated with scores on the STAI (Spearman's $\rho=0.58, d f=16, p<0.03$ ).

\section{Discussion}

In this pilot report, we provide evidence that neurochemical changes in dopaminergic, serotonergic, and opioid systems may be associated with the clinical expression of dissociation in patients with eating disorders during the acute phase of their illness. While these potential relationships are evident in the overall group of eating-disordered patients studied here, they appear more specific for the subset of patients with normal-weight bulimia nervosa, and are not seen in the separate analysis of the underweight anorexic subjects. Our data also replicate the earlier work of Kaye et al. $(1987,1988)$ demonstrating a reduction in CSF levels of $\beta$ endorphin and the serotonin metabolite 5-HIAA in underweight patients with anorexia nervosa compared with normal-weight women. The causes and implications of the observations reported here are unknown. Nevertheless, they raise several points that deserve comment and may help highlight areas for future study.

Malnutrition is associated with a spectrum of biochemical and psychopathological sequelae. Indeed, metabolic evidence of starvation may be detected long before a substantial change in body weight occurs (Pirke et al., 1985). Hence, despite the fact that total DES scores do not segregate by diagnostic group, it may be argued that the relationships described here represent the nonspecific confluence of such starvationrelated events. On the other hand, the biological and behavioral associations were more apparent in the normal-weight bulimic patients than in the underweight anorexic patients, although the former group arguably represents the less nutritionally impaired of the two patient populations. Moreover, when the eating disorder group was examined in aggregate, there was no association between the total DES score and any measure of illness chronicity or severity (i.e., clinical measures that could be expected to reflect, from various perspectives, the relative nutritional compromise of the individual). These considerations would suggest that the associations between the CSF neurochemicals and DES scores reported here may not be a simple reflection of the nutritional impairment associated with the eating disorders themselves. In other words, the clinical expression of dissociative experience is an overdetermined event. Chronic malnutrition, through food restriction or episodes of binge eating and purging, may exert a permissive, or amplifying, effect and may therefore be a necessary but not sufficient condition for the full expression of dissociative phenomena in patients with eating disorders. More specific metabolic measures of starvation obtained concurrently with assessment of dissociative capacity may be helpful in clarifying this point in future studies.

Dissociative events are known to produce psychobiologically distinct states of consciousness across alter personalities in patients with multiple personality disorder that differ significantly from those seen in simulating control subjects. Support for 
this assertion arises from studies that have included examination of spontaneous electroencephalographic activity, evoked potential responses, and autonomic nervous system reactivity in this clinical population (Putnam et al., 1990; Putnam, $1991 b$ ). Furthermore, patients with multiple personality disorder have been shown to demonstrate significant disruptions in state-dependent learning and retrieval across alter personalities, as well as significantly greater variability of visual functioning than normal control subjects who are role-playing different personalities (Silberman et al., 1985; Nissen et al., 1988; Miller, 1989; Schacter et al., 1989; Miller et al., I991; Putnam, 1991b).

Despite these considerations, little is known about the underlying neurochemical correlates of dissociative phenomena. It has been suggested that a clue to the neurobiology of dissociation may be inferred from the observation that the transition from one alter personality to another (referred to as "switching") that occurs in patients with multiple personality disorder resembles a number of other mood-state transition phenomena seen in psychiatric disorders such as panic attacks, mood cycling in bipolar patients, and dramatic mood shifts in patients with borderline personality disorder (Putnam, 1988). Hence, these events may share common pathophysiologic mechanisms. Switching in patients with multiple personality disorder is often associated with a triggering event that has particular psychological relevance to the individual. This latter observation is of interest to the present report in light of the well-established association between childhood trauma and adult dissociative disorders (Putnam, 1985), and the increasing evidence of traumatic antecedents, primarily childhood sexual abuse, in a substantial percentage of patients with eating disorders (Damlouji and Ferguson, 1985; Goldfarb, 1987; Schechter et al., 1987; Torem and Curdue, 1988; Coons et al., 1989; Folsom et al., 1989). The specific etiologic significance of such traumatic childhood abuse for the development of eating disorders is doubtful (Pope and Hudson, 1992). Nevertheless, clinical observation suggests that exacerbations of both dissociative disorders and eating disorders in patients with a history of abuse may be provoked by internal or external triggers contextually similar to the historical trauma (e.g., emergence of sexual feelings during pubertal development or establishment of adult sexual relationships).

The negative correlation between CSF $\beta$-endorphin and a range of behavioral measures (DES, BDI, and STAI) in the overall patient group raises the theoretical possibility that CNS opioids may be an important participant in the stabilization of psychological states or, at least, in diminishing the propensity for a dissociative shift from one state to another. It is attractive to hypothesize that a putative dysregulation in the endogenous opiate system in patients with increased levels of dissociative experience may also relate to the distortions in pain perception (Lautenbacher et al., 1991) and the clinically evident propensity toward self-harmful behavior seen in these individuals (Demitrack et al., 1990). Although no significant association was found between a history of self-harm and any of the biological measures reported here, it was of interest that CSF levels of $\beta$-endorphin were nonsignificantly lower in those patients with a history of self-harmful behavior $(1.98 \pm 1.49$ vs. $3.14 \pm 2.66$ $\mathrm{fmol} / \mathrm{ml}$, two-tailed unpaired $t$ test, $t=-1.278, d f=22, p=0.21$ ). 
Further evidence that the present findings may have more general relevance for an understanding of the neurobiology of dissociation is provided by a recent report from Spiegel and King (1992), who described a positive correlation between hypnotizability and CSF levels of HVA in a non-eating-disordered population, similar in magnitude to the relationship between total DES score and HVA described in the present report. Hypnosis is a phenomenon that shares many clinical and experimental features of, but is not synonymous with, dissociation (Carlson and Putnam, 1989; Putnam, 1991a). Spiegel and his collaborators concluded that dopaminergic neurotransmission, especially involving functional activation of frontal lobe pathways, may be an important neurobiologic substrate for the generation of hypnotic phenomena. Such a view is compatible with the neodissociation model of hypnotic experience described by Hilgard (1986), wherein hypnosis may alter conscious awareness by impinging on a central, executive control system (e.g., frontal lobes) which itself coordinates the activity of several hierarchically arranged cognitive subsystems. Pettinati et al. (1985) have previously reported that patients with eating disorders are highly hypnotizable. In that report, the increase in hypnotizability was largely due to substantial elevations in hypnotic capacity among normal-weight bulimic patients and underweight anorexic patients with bulimic features. Although DES scores did not differ in the anorexic and bulimic subgroups in the present study, it should be emphasized that the anorexic sample was predominantly represented by underweight bulimic anorexic patients ( 8 of 11 subjects). Hence, the disparity between our findings and those of Pettinati et al. may be more apparent than real. Further work with a larger, more heterogeneous sample appears warranted.

In summary, the preliminary results in this report are consistent with previous studies of biochemical disturbances in patients with eating disorders and are also compatible with recent findings concerning the related phenomenon of hypnosis. Furthermore, these data suggest that future work in the psychobiology of dissociation should target a more detailed examination of these neurochemical systems.

Acknowledgments. The authors are grateful to William Ericson, Ph.D., for help with the statistical analysis of the data contained in this article, and Ms. Candy Davis, for performing the $\beta$-endorphin assays.

\section{References}

American Psychiatric Association. DSM-III-R: Diagnostic and Statistical Manual of Mental Disorders. 3rd ed., revised. Washington, DC: American Psychiatric Press, 1987. p. 269.

Beck, A.T.; Ward, C.H.; Mendelson, M.; Mock, J.; and Erbaugh, J. An inventory for measuring depression. Archives of General Psychiatry, 5:561-571, 1961.

Bernstein, E., and Putnam, F. Development, reliability and validity of a dissociation scale. Journal of Nervous and Mental Disease, 174:727-735, 1986.

Carlson, E.B., and Putnam, F.W. Integrating research on dissociation and hypnotizability: Are there two pathways to hypnotizability? Dissociation, 2:32-38, 1989.

Coons, P.M.; Bowman, E.S.; Pellow, T.A.; and Schneider, P. Post-traumatic aspects of the treatment of victims of sexual abuse and incest. Psychiatric Clinics of North America, 12:325$335,1989$. 
Damlouji, N.F., and Ferguson, J.M. Three cases of posttraumatic anorexia nervosa. American Journal of Psychiatry, 142:362-363, 1985.

Demitrack, M.A.; Putnam, F.W.; Brewerton, T.D.; Brandt, H.A.; and Gold, P.W. Relation of clinical variables to dissociative phenomena in eating disorders. American Journal of Psychiatry, 147:1184-1188, 1990.

Folsom, M.S.; Krahn, D.D.; Nairn, K.; Gold, L.; and Silk, K. Sex abuse: Role in eating disorders. Annual Meeting of the American Psychiatric Association, Research Program and Abstracts. Washington, DC: APA, 1989. p. 203.

Goldfarb, L.A. Sexual abuse antecedent to anorexia nervosa, bulimia, and compulsive overeating: Three case reports. International Journal of Eating Disorders, 6:665-680, 1987.

Hilgard, E.R. Divided Consciousness: Multiple Controls in Human Thought and Action. New York: John Wiley \& Sons, 1986.

Kaye, W.H.; Berrettini, W.H.; Gwirtsman, H.E.; Chretien, M.; Gold, P.W.; George, D.T.; and Jimerson, D.C. Reduced cerebrospinal fluid levels of immunoreactive pro-opiomelanocortin peptides (including beta-endorphin) in anorexia nervosa. Life Sciences, 41:2147-2155, 1987.

Kaye, W.H.; Gwirtsman, H.E.; George, D.T.; Jimerson, D.C.; and Ebert, M.H. CSF 5HIAA concentrations in anorexia nervosa: Reduced values in underweight subjects normalize after weight gain. Biological Psychiatry, 23:102-105, 1988.

Lautenbacher, S.; Pauls, A.M.; Strian, F.; Pirke, K.-M.; and Krieg, J.-C. Pain sensitivity in anorexia nervosa and bulimia nervosa. Biological Psychiatry, 29:1072-1078, 1991.

Miller, S. Optical differences in cases of multiple personality disorder. Journal of Nervous and Mental Disease, 177:480-486, 1989.

Miller, S.D.; Blackburn, T.; Scholes, G.; White, G.L.; and Mamalis, N. Optical differences in multiple personality disordcr: A second look. Journal of Nervous and Mental Disease, 179:132-135, 1991.

Nissen, M.J.; Ross, J.L.; Willingham, D.B.; Mackenzie, T.B.; and Schacter, D.L. Memory and awareness in a patient with multiple personality disorder. Brain and Cognition, 8:117-134, 1988.

Pettinati, H.M.; Horne, R.L.; and Staats, J.M. Hypnotizability in patients with anorexia nervosa and bulimia. Archives of General Psychiatry, 42:1014-1016, 1985.

Pirke, K.M.; Pahl, J.; Schweiger, U.; and Warnhoff, M. Metabolic and endocrine indices of starvation in bulimia: A comparison with anorexia nervosa. Psychiatry Research, 15:33-39, 1985.

Pope, H.G., Jr., and Hudson, J.I. Is childhood sexual abuse a risk factor for bulimia nervosa? American Journal of Psychiatry, 149:455-463, 1992.

Putnam, F.W. Dissociation as a response to extreme trauma. In: Kluft, R.P., ed. Childhood Antecedents of Multiple Personality. Washington, DC: American Psychiatric Press, 1985. pp. 66-97.

Putnam, F.W. The switch process in multiple personality disorder and other state-change disorders. Dissociation. 1:24-32, 1988.

Putnam, F.W. Dissociative phenomena. In: Tasman, A., ed. Annual Review of Psychiatry. Washington, DC: American Psychiatric Press, 1991a. pp. 159-174.

Putnam, F.W. Recent research on multiple personality disorder. Psychiatric Clinics of North America, 14:489-502, $1991 b$.

Putnam, F.; Zahn, T.; and Post, R. Differential autonomic nervous system activity in multiple personality disorder. Psychiatry Research, 31:251-260, 1990.

Quimby, L.C., and Putnam, F.W. Dissociative symptoms and aggression in a state mental hospital. Dissociation, 4:21-24, 1991.

Ross, C.A., and Norton, G.R. Suicide and parasuicide in mulitiple personality disorder. Psychiatry, 52:365-371, 1989. 
Schacter, D.L.; Kihlstrom, J.F.; Kihlstrom, L.C.; and Berren, M.B. Autobiographical memory in a case of multiple personality disorder. Journal of Abnormal Psychology, 98:1-7, 1989.

Schechter, J.O.; Schwartz, H.P.; and Greenfeld, D.G. Sexual assault and anorexia nervosa. International Journal of Eating Disorders, 5:313-316, 1987.

Seppala, T.; Scheinin, M.; Capone, A.; and Linnoila, M. Liquid chromatographic assay for CSF catecholamines using electrochemical detection. Acta Pharmacologica et Toxicologica. 55:81-97, 1984.

Silberman, E.K.; Putnam, F.W.; Weingartner, H.; Braun, B.G.; and Post, R.M. Dissociative states in multiple personality disorder: A quantitative study. Psychiatry Research, 15:253-260, 1985.

Spiegel, D. Dissociation and trauma. In: Tasman, A., and Goldfinger, S.M., eds. Annual Review of Psychiatry. Washington, DC: American Psychiatric Press, 1991. pp. 261-266.

Spiegel, D., and King, R. Hypnotizability and CSF HVA levels among psychiatric patients. Biological Psychiatry, 31:95-98, 1992.

Spielberger, C.D.; Gorsuch, R.L.; and Lushene, R.D. STAI Manual. Palo Alto, CA: Consulting Psychologists Press, 1970.

Torem, M., and Curdue, K. PTSD presenting as an eating disorder. Stress Medicine, 4:139-142, 1988.

World Health Organization. Mental Disorders: Glossary and Guide to Their Classification in Accordance With the Ninth Revision of the International Classification of Diseases. Geneva: WHO, 1978. 